\title{
Étude des performances de machines-outils 5 axes à structure parallèle et sérielle pour l'usinage d'une pièce aéronautique
}

\author{
Sylvain Pateloup, Hélène Chanal a et Emmanuel DuC \\ Clermont Université, IFMA, EA 3867, Laboratoire de Mécanique et Ingénierie, BP 10448, 63000 Clermont-Ferrand, France
}

Reçu le 6 janvier 2010, accepté le 9 juin 2011

\begin{abstract}
Résumé - L'utilisation de machines-outils à structure parallèle devrait permettre d'accroître la productivité de l'usinage par rapport aux machines-outils à structure sérielle pour des performances cinématiques des axes similaires. Ces machines-outils doivent cependant présenter une rigidité de leur structure, une précision de leur commande, et une accessibilité d'outil suffisantes pour l'usinage de pièces aéronautiques. Cet article propose alors de comparer les performances durant l'usinage d'une machine-outil 5 axes à structure parallèle, la Tripteor X7 de PCI, et d'une machine-outil 5 axes à structure sérielle, la KX15 de Huron. La comparaison est réalisée à l'aide d'une pièce test représentative du secteur aéronautique. Les paramètres évalués sont le temps d'usinage pour les différentes opérations, la qualité dimensionnelle et géométrique de la pièce réalisée et l'état de surface des surfaces obtenues. Ces essais illustrent le potentiel des machines-outils à structure parallèle à usiner des pièces aéronautiques avec une qualité dimensionnelle et géométrique satisfaisante en augmentant la productivité. Cependant, la qualité d'état de surface obtenue avec la machine-outil à structure parallèle PCI Tripteor X7 n'est pas acceptable vis-à-vis des contraintes de qualité des pièces aéronautiques. Ainsi, plusieurs voies d'amélioration du processus d'usinage avec les machines-outils à structure parallèle sont proposées afin de mieux maîtriser la productivité et l'état de surface obtenue durant l'usinage.
\end{abstract}

Mots clés : Machines-outils à structure parallèle / comparaison / productivité / qualité d'usinage

\begin{abstract}
A study of the performances of parallel and serial 5 axis machine-tools for aeronautics part machining. Parallel kinematic machine-tools (PKM) allows improving machining productivity compared to serial kinematic machine-tools (SKM) having the same kinematic performances of axis. However, PKM have to offer sufficient structure stiffness, command accuracy and machining accessibility to be used in aeronautics machining tasks. This paper proposes to compare machining performances of a PKM, the Tripteor X7 designed by PCI and a SKM, the KX15 designed by Huron. The comparison is done for the machining of a test part having the main characteristics of aeronautics sector. Evaluated parameters are machining time, dimensional, geometric and roughness quality. These experiments show the potential of PKM to machine aeronautic parts with an accepted dimensional and geometric quality and a higher productivity. However, they highlight a non acceptable roughness with regard to the quality constraints of aeronautics products. Thus, several improvement ways of the machining quality with PKM are proposed in order to better predict and control the obtained machining productivity and quality.
\end{abstract}

Key words: Parallel kinematic machine-tool / comparison / productivity / machining quality

\section{Introduction}

Depuis une dizaine d'années, des machines d'usinage à grande vitesse (UGV) à structure parallèle apparaissent dans l'industrie automobile et aéronautique, car elles présentent de meilleures performances dynamiques que les machines-outils à structure sérielle, pour un même

\footnotetext{
a Auteur pour correspondance : helene.chanal@ifma.fr
}

comportement cinématique des axes motorisés [1]. Toutefois, elles offrent une précision d'usinage dégradée, principalement à cause d'une rigidité plus faible de la structure, et des erreurs de transformation géométrique plus grandes [2]. Ainsi, ces machines sont principalement utilisées pour l'usinage en grande série de pièces préformées, car elles offrent une grande accessibilité, une forte productivité et une qualité d'usinage suffisante [3]. Certaines 


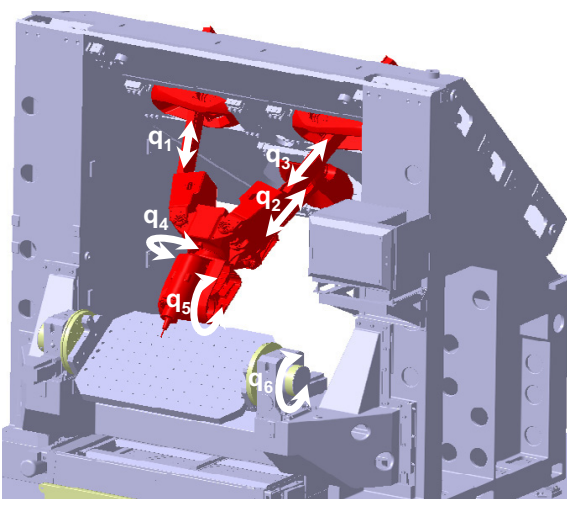

$\mathrm{PCl}$ Tripteor X7

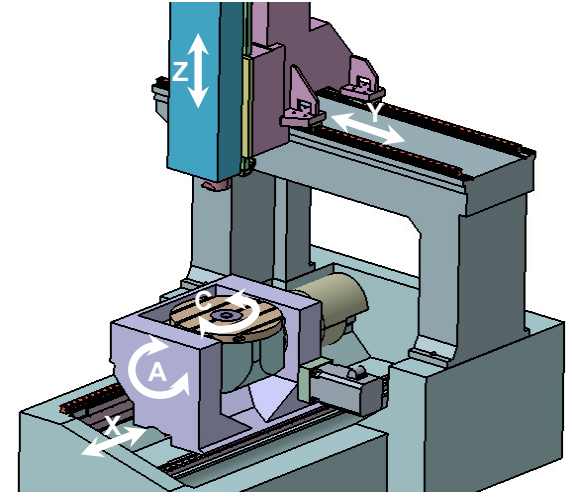

Huron KX15

Fig. 1. Architecture de la PCI Tripteor X7 (gauche) et de la Huron KX15 (droite).

machines-outils à structure parallèle, comme l'Ecospeed de DS-Technologie, sont également utilisées pour l'usinage de pièces de structure aéronautique, car le parallélisme des axes permet d'augmenter la vitesse de changement d'orientation de l'outil [4]. La conception de ces machines-outils privilégie une augmentation de la rigidité des éléments et de la structure au détriment de l'accessibilité. Ainsi, une nouvelle architecture parallèle de machineoutil, l'Exechon, a été développée de façon à concilier rigidité et accessibilité $[5,6]$.

Il est alors nécessaire d'évaluer et de qualifier, en termes de productivité et de qualité d'usinage, ces nouvelles machines-outils à structure parallèle en fonction de l'application d'usinage visée. Cet article présente une étude comparative des performances d'une machine-outil 5 axes à structure parallèle et d'une machine-outil 5 axes à structure sérielle pour l'usinage d'une pièce aéronautique nécessitant un fort taux d'enlèvement de matière et une qualité finale conforme aux spécifications. L'objectif de l'étude est d'analyser les différences de précision de pose de l'outil, de comportement mécanique et dynamique de la structure des deux machines-outils en comparant les temps et les défauts d'usinage.

Dans un premier temps, cet article présente les machines-outils ainsi que la pièce retenue pour la qualification. Ensuite, les résultats comparatifs de temps d'usinage, défauts dimensionnels, défauts géométriques et défauts d'état de surface sont analysés pour qualifier le comportement des machines-outils durant l'usinage. Plusieurs voies d'amélioration du comportement de ces machines sont alors étudiées en observant leur influence sur la productivité et la qualité de l'usinage.

\section{Présentation des machines-outils utilisées pour les essais comparatifs}

L'étude comparative est appliquée sur la machineoutil à structure parallèle Tripteor X7 de PCI-SCEMM, et la machine-outil à structure sérielle KX15 de Huron (Fig. 1)
La machine-outil PCI Tripteor X7 met en ouvre le robot Exechon à tête orientable et possède un $6^{\mathrm{e}}$ axe positionné permettant d'orienter la table et la pièce. La machine-outil Huron KX15 est une machine outil à 5 axes possédant une table à architecture plateau-berceau de type A-C. Ses propriétés de rigidité et de précision la rendent performante pour l'usinage de moules industriels. Les deux machines-outils présentent des caractéristiques similaires au niveau de l'espace de travail, de la commande numérique $(\mathrm{CN})$ et des performances cinématiques et dynamiques des axes en translation (Tab. 1). Les programmes d'usinage sont identiques. Ainsi, la différence de performance de l'usinage provient principalement du comportement de la structure de la machine, des éventuelles erreurs de transformation géométrique inverse [2]. Les performances réduites des axes de rotation de la machineoutil sérielle, liées à son architecture de type berceauplateau, peuvent également pénaliser la productivité pour des opérations nécessitant des changements d'orientation de l'outil.

\section{Performances observées durant l'usinage d'une pièce test}

La pièce utilisée est une cornière d'assemblage pour l'aéronautique, possédant un ratio de volume entre la pièce usinée et le brut d'usinage de 1/16. Les entités à usiner sont décrites sur la figure 2. Les opérations d'usinage d'ébauche sont réalisées en usinage 5 axes positionnés avec des débits d'enlèvements de matière importants. Les opérations de fraisage de finition sont réalisées soit en usinage 5 axes positionnés par contournage ou balayage 2 axes $1 / 2$, soit en usinage 5 axes continus avec un unique outil torique. Cette pièce permet ainsi d'étudier le comportement des machines-outils pour l'usinage 2 axes $1 / 2$ d'entités élémentaires et de formes complexes $[1,7]$. Elle offre également la possibilité d'analyser le comportement des machines-outils durant l'usinage en 5 axes continus. Enfin, cette pièce permet d'observer l'accessibilité offerte par la machine-outil et la rapidité des déplacements 
Tableau 1. Performances cinématiques et dynamiques des axes des machines-outils.

\begin{tabular}{|c|c|c|c|c|c|c|}
\hline & \multicolumn{2}{|c|}{ Vitesse maximale } & \multicolumn{2}{|c|}{ Accélération maximale } & \multicolumn{2}{|c|}{ Jerk maximal } \\
\hline & PCI & Huron & PCI & Huron & PCI & Huron \\
\hline & Tripteor X7 & KX15 & Tripteor X7 & KX15 & Tripteor X7 & KX15 \\
\hline Axes de translation & $22 \mathrm{~m} \cdot \mathrm{min}^{-1}$ & $30 \mathrm{~m} \cdot \mathrm{min}^{-1}$ & $2,5 \mathrm{~m} \cdot \mathrm{s}^{-2}$ & $3 \mathrm{~m} \cdot \mathrm{s}^{-2}$ & $40 \mathrm{~m} \cdot \mathrm{s}^{-3}$ & $30 \mathrm{~m} \cdot \mathrm{s}^{-3}$ \\
\hline Axes de rotation & 45 tr.min $^{-1}$ & 20 tr.min $^{-1}$ & 6 tr.s ${ }^{-2}$ & 0,75 tr.s $\mathrm{s}^{-2}$ & 150 tr.s $\mathrm{s}^{-3}$ & $140(\mathrm{C}) 50(\mathrm{~A})$ tr.s $^{-3}$ \\
\hline
\end{tabular}

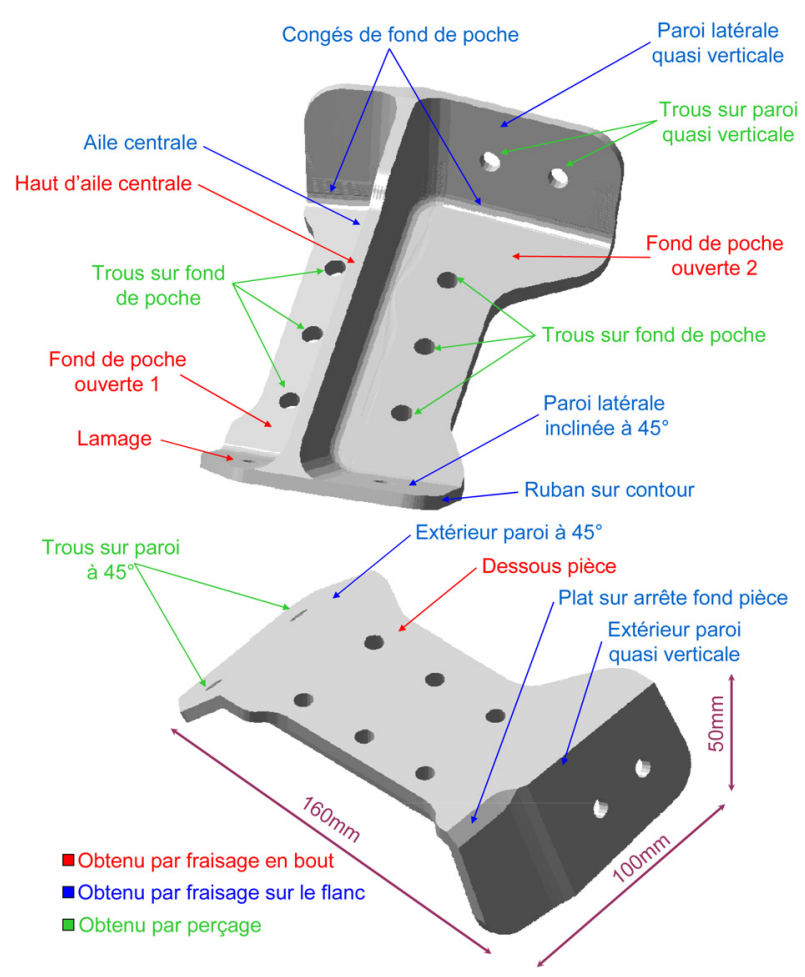

Fig. 2. Morphologie de la pièce test et entités à usiner.

hors-matières entre les opérations et durant les opérations d'ébauche [3].

L'évaluation de la performance de chaque machine se fait à travers le relevé du temps d'usinage, la mesure des défauts géométriques et dimensionnels des entités usinées et la mesure de rugosité. Seule la première phase d'usinage est évaluée (Fig. 3), pour ne pas prendre en compte les défauts de remise en position de la pièce. Les performances d'usinage de chaque machine sont étudiées pour chaque catégorie d'opération classées vis-à-vis des sollicitations générées par la commande et la structure de la machine-outil.

Ainsi, les opérations d'ébauche permettent de qualifier plus particulièrement la productivité pour des trajectoires 2 axes $1 / 2$ comprenant des trajets rapides (G0) avec des valeurs élevées des paramètres dynamiques. Les opérations de finition, utilisant des valeurs faibles des paramètres dynamiques permettent d'isoler l'influence de la structure de la machine-outil sur la productivité, les défauts dimensionnels et la rugosité des surfaces usinées. Il faut distinguer les opérations de finition 3 axes, qui ne sollicitent qu'une partie des axes pour la machine-outil sérielle, des opérations de finition 5 axes continus, sollicitant l'ensemble des axes des deux machines-outils.

\section{Analyse des résultats}

Le temps d'usinage est mesuré par comptage du nombre de périodes d'interpolation (correspondant à un temps de $4 \mathrm{~ms}$ pour la CN Siemens 840D équipant les deux machines-outils) écoulées durant l'exécution du programme d'usinage. La PCI Tripteor X7 procure un gain de productivité global de 11 \% (Tab. 2), alors qu'elle possède des performances de vitesse et d'accélération d'axes en translation inférieures de $25 \%$ et $15 \%$ respectivement. En effet, le facteur d'amplification cinématique dû à la structure parallèle permet un gain de vitesse de déplacement de l'outil par rapport aux déplacements des axes motorisés $[8,9]$.

Ce facteur d'amplification cinématique accroît le gain de productivité pour des trajets rapides plus nombreux dans les opérations d'ébauche (9\% pour les opérations d'ébauche contre $7 \%$ pour les opérations de finition). Il permet aussi d'obtenir des changements d'orientation de l'outil plus rapides qui sollicitent plus en amplitude les axes de translation (plus dynamiques) et moins les axes de rotation (moins dynamiques), tout en conservant une accessibilité suffisante. Un gain de productivité de $20 \%$ est ainsi atteint pour les opérations d'usinage à 5 axes sur le flanc. Cette valeur importante résulte également de la différence des performances dynamiques des axes rotatifs entre les deux machines-outils. Ces axes rotatifs sont, pour la Huron KX15, sollicités en permanence pour les opérations en 5 axes continus alors qu'ils ne le sont que durant moins de $1 \%$ du temps d'usinage pour les opérations en 5 axes positionnés.

Les défauts dimensionnels (mesurés avec une machine à mesurer multi-capteurs) et géométriques (mesurés avec une machine à mesurer tridimensionnelle) des entités usinées et repérées figure 3 sont présentés dans le tableau 3. L'ensemble des défauts mesurés sont exprimés en $\mu \mathrm{m}$, comme le spécifie la norme de cotation par spécifications géométriques des produits (GPS).

Les défauts dimensionnels et d'orientation proviennent principalement des erreurs statiques de pose de l'outil (transformation géométrique inverse, compensation des déformations dues aux forces de gravité et appliquées au chargement thermique) appliquées à la cellule d'usinage. Les défauts de forme sont des défauts intrinsèques à la surface usinée provenant du comportement de la structure (déformations, vibrations) et des erreurs de suivi provoquées par la commande [2]. Ils 
Tableau 2. Temps d'usinage mesurés.

\begin{tabular}{|c|c|c|c|c|}
\hline Temps d'usinage & $\begin{array}{c}\text { Simulation } \\
\text { FAO }\end{array}$ & $\begin{array}{c}\text { PCI } \\
\text { Tripteor X7 }\end{array}$ & $\begin{array}{l}\text { Huron } \\
\text { KX15 }\end{array}$ & $\begin{array}{c}\text { Gain de productivité } \\
\text { avec la Tripteor X7 }\end{array}$ \\
\hline $\begin{array}{l}\text { Ébauche en } 5 \text { axes positionnés } \\
\text { (jerk maximum : } 20 \mathrm{~m} . \mathrm{s}^{-3} \text { pour axes } 1,2,3 \\
\text { et } 80 \mathrm{tr} . \mathrm{s}^{-3} \text { pour axes } 4,5 \text { ) }\end{array}$ & $432 \mathrm{~s}$ & $517 \mathrm{~s}$ & $567 \mathrm{~s}$ & $9 \%$ \\
\hline $\begin{array}{c}\text { Finition en } 5 \text { axes positionnés } \\
\text { (jerk maximum : } 5 \mathrm{~m} \cdot \mathrm{s}^{-3} \text { pour axes } 1,2,3 \\
\left.\text { et } 20 \mathrm{tr} . \mathrm{s}^{-3} \text { pour axes } 4,5\right)\end{array}$ & $282 \mathrm{~s}$ & $403 \mathrm{~s}$ & $435 \mathrm{~s}$ & $7 \%$ \\
\hline $\begin{array}{l}\text { Finition en } 5 \text { axes continus } \\
\text { (jerk maximum : } 5 \mathrm{~m} \cdot \mathrm{s}^{-3} \text { pour axes } 1,2,3 \\
\left.\text { et } 20 \mathrm{tr} . \mathrm{s}^{-3} \text { pour axes } 4,5\right)\end{array}$ & $96 \mathrm{~s}$ & $243 \mathrm{~s}$ & $302 \mathrm{~s}$ & $20 \%$ \\
\hline
\end{tabular}

Tableau 3. Défauts dimensionnels et géométriques mesurés.

\begin{tabular}{|c|c|c|c|c|}
\hline $\begin{array}{l}\text { Classe de } \\
\text { défaut }\end{array}$ & Type de défaut & $\begin{array}{c}\text { PCI } \\
\text { Tripteor X7 }\end{array}$ & $\begin{array}{l}\text { Huron } \\
\text { KX15 }\end{array}$ & $\begin{array}{c}\text { Évolution des défauts } \\
\text { avec la Tripteor X7 }\end{array}$ \\
\hline \multirow{7}{*}{ Défauts dimensionnels $(\mu \mathrm{m})$} & Distance trou $1 /$ trou 2 & 10 & 6 & \multirow{7}{*}{$\times 2$} \\
\hline & Distance trou $3 /$ trou 2 & 6 & 12 & \\
\hline & Distance trou $4 /$ trou 5 & 10 & 2 & \\
\hline & Distance trou $6 /$ trou 5 & 3 & 5 & \\
\hline & Distance trou 4/trou 1 & 36 & 3 & \\
\hline & Distance trou $5 /$ trou 2 & 30 & 15 & \\
\hline & Distance trou $6 /$ trou 3 & 23 & 10 & \\
\hline \multirow{8}{*}{ Défauts de forme $(\mu \mathrm{m})$} & Planéité plan 1 & 11 & 7 & \multirow{8}{*}{$\times 1,5$} \\
\hline & Planéité plan 2 & 18 & 11 & \\
\hline & Planéité plan 3 & 17 & 11 & \\
\hline & Planéité plan 4 & 16 & 15 & \\
\hline & Planéité plan 5 & 10 & 6 & \\
\hline & Planéité plan 6 & 16 & 6 & \\
\hline & Planéité plan 7 & 8 & 7 & \\
\hline & Planéité plan 8 & 12 & 12 & \\
\hline \multirow{4}{*}{ Défauts d'orientation $(\mu \mathrm{m})$} & Inclinaison plan $2 /$ plan $1\left(10^{\circ}\right)$ & 18 & 14 & \multirow{4}{*}{$\times 1,5$} \\
\hline & Inclinaison plan $3 /$ plan $1\left(95^{\circ}\right)$ & 7 & 5 & \\
\hline & Inclinaison plan 5/plan $1\left(45,75^{\circ}\right)$ & 10 & 7 & \\
\hline & Inclinaison plan $7 /$ plan $1\left(24,90^{\circ}\right)$ & 9 & 7 & \\
\hline
\end{tabular}

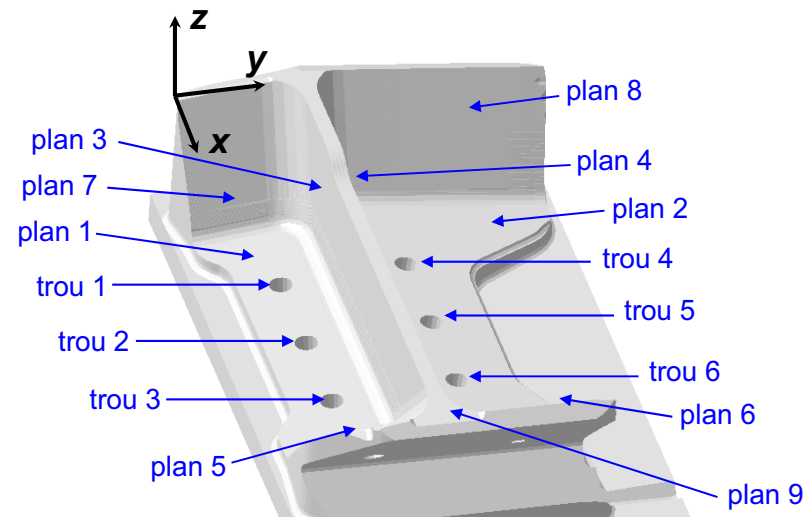

Fig. 3. Entités géométriques mesurées suite à la première phase d'usinage de la pièce test.

proviennent également de l'évolution des erreurs statiques pour la machine-outil à structure parallèle à cause de son comportement anisotrope [9].
Ainsi, les défauts dimensionnels mesurés sont en moyenne 2 fois plus élevés pour la pièce usinée avec la PCI Tripteor X7, tandis que les défauts de forme et d'orientation sont en moyenne 1,5 fois plus importants. En effet, l'usinage de cette pièce met en évidence l'influence des erreurs d'ordres statique et dynamique de la structure de la PCI Tripteor X7. Toutefois, les valeurs des défauts géométriques mesurés sont compatibles avec des applications du secteur aéronautique. De plus, les défauts dimensionnels mesurés sont plus élevés suivant la direction d'usinage $\boldsymbol{y}$ que suivant la direction $\boldsymbol{x}$, du fait du comportement statique anisotrope de la structure de la machine-outil [10]. Les défauts de formes varient peu entre les entités contrôlées. Ainsi, l'anisotropie du comportement mécanique de la structure influence peu la qualité géométrique de la pièce dans la zone de l'espace de travail utilisée pour l'usinage de chaque entité.

Les défauts d'état de surface, résultant du comportement dynamique de la cellule d'usinage, sont évalués par la rugosité arithmétique $(R a)$ et la rugosité totale par longueur d'évaluation de la mesure $(R z)$, mesurées dans 


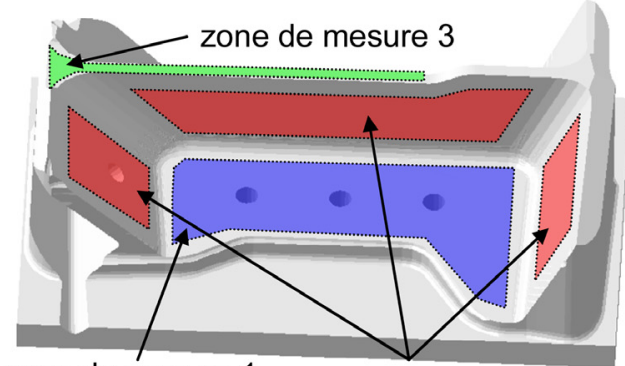

zone de mesure 1

zone de mesure 2

Mesure des états de surfaces

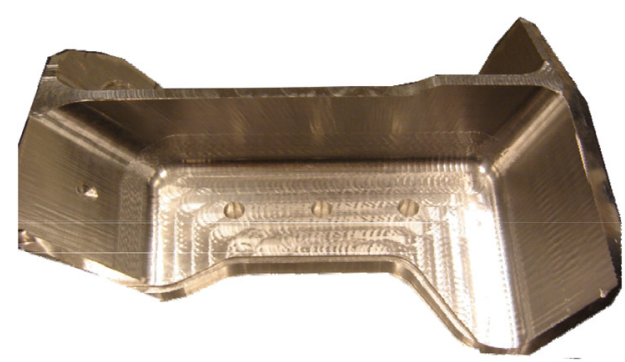

Pièce usinée avec la Tripteor X7

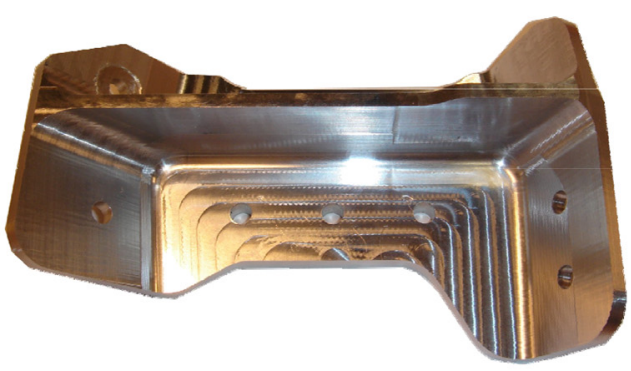

Pièce usinée avec la KX 15

Fig. 4. Visualisation et mesure des défauts d'état de surface sur la pièce test.

différentes zones avec un rugosimètre à contact unidimensionnel (Fig. 4).

Le tableau 4 présente les valeurs de rugosité maximum mesurées dans une direction tangente à la trajectoire, sur laquelle se répercutent les différentes erreurs dynamiques étudiées lors de ces essais. Les défauts d'ondulations sont plus importants sur la pièce usinée avec la PCI Tripteor $\mathrm{X} 7$, ce qui dégrade la valeur de $R z$ (Fig. 3).

Les défauts d'état de surface générés sont maximaux avec la PCI Tripteor X7 et ne sont pas acceptables vis-àvis des exigences de l'industrie aéronautique. Par ailleurs, la rugosité mesurée est maximale pour les deux pièces usinées au niveau des discontinuités en tangence ou en courbure des trajectoires d'usinage et pour les trajectoires en 5 axes continus. Le comportement mécanique de la structure parallèle génère plus de défauts lors du passage d'une discontinuité de la trajectoire d'usinage.

Ces essais comparatifs montrent que les performances de la PCI Tripteor X7 varient suivant la position et la direction de déplacement de l'outil. Ainsi, le gain en productivité est différent pour toutes les opérations. Il en est de même pour la dégradation de l'état de surface amplifiée pour certaines portions du trajet d'outil (Fig. 4). Ces observations justifient la mise en place d'une analyse supplémentaire du processus d'usinage pour les machinesoutils à structure parallèle de façon à améliorer leur comportement pendant l'usinage.

\section{Vers une amélioration du processus d'usinage sur machine-outil à structure parallèle}

Une amélioration de l'état de surface peut apparaître en remplaçant le format de description linéaire des trajectoires d'usinage (format G1) par un format de description polynomial (format B-spline Siemens par exemple) [11]. En effet, les discontinuités en tangence induites aux raccordements des segments par le format G1 entraînent des sauts d'accélération pouvant exciter la structure selon ses modes propres [12]. Le format B-spline Siemens (B-spline cubique) procure une continuité $\mathrm{C}^{2}$ de la trajectoire décrite dans le programme d'usinage et décharge alors le DCN de certains calculs d'interpolation et de lissage de la trajectoire en temps réel, dont l'efficacité est parfois limitée [11].

$\mathrm{Si}$ ce format de description n'apporte pas d'amélioration notable de l'état de surface obtenu avec la Huron KX15, il permet une diminution de la rugosité (jusqu'à $40 \%$ ) pour l'usinage réalisé avec la PCI Tripteor X7. Il procure également une augmentation de la productivité des deux machines-outils $(+10 \%$ pour l'opération d'usinage sur le flanc 5 axes réalisée avec la PCI Tripteor X7 avec un temps d'usinage de 220 s contre $243 \mathrm{~s})$. Cependant, les gains apportés par ce format de description ne sont pas encore suffisants pour atteindre la qualité d'état de surface procurée par la Huron KX15 (Tab. 5).

Les défauts d'ondulation (apparaissant avec une fréquence de $20 \mathrm{~Hz}$ ) restent importants et semblent provenir de vibrations régénératives de la structure de la machine-outil sollicitée par le mouvement des axes et le phénomène de coupe [13-15]. En effet, l'analyse des relevés de jerk des axes fait apparaître des valeurs importantes au cours de l'usinage en 5 axes continus (Fig. 5). Elles peuvent atteindre jusqu'à 4 fois la valeur du paramètre de limitation de jerk articulaire renseignée dans le DCN (paramètres Siemens 32431 et 32432 ). Pour les machines-outils à structure parallèle, ce phénomène peut provenir d'une incohérence de traitement de la trajectoire par le DCN qui adapte théoriquement les consignes des moteurs des axes au paramétrage des contraintes dynamiques (vitesse, accélération et jerk) s'exerçant à la fois sur l'outil et sur les axes [11].

Une autre origine de ces défauts peut être liée aux variations de jerk, appelées snap (dérivée temporelle $4^{\mathrm{e}}$ de la position), trop brutales. En effet, ce paramètre n'est pas contrôlé lors de la génération de trajectoires B-spline cubiques et peut provoquer des vibrations lorsque la valeur 
Tableau 4. Rugosités mesurées.

\begin{tabular}{cccccc}
\hline & \multicolumn{2}{c}{ PCI Tripteor X7 } & \multicolumn{2}{c}{ Huron $\mathrm{KX} 15$} & \multirow{2}{*}{ Évolution de $R z$ avec la Tripteor X7 } \\
\cline { 1 - 4 } Rugosité $(\mu \mathrm{m})$ & $R a_{\max }$ & $R z_{\max }$ & $R a_{\max }$ & $R z_{\max }$ & $\times 5,4$ \\
\cline { 1 - 3 } Zone de mesure 1 & 4,1 & 17,2 & 0,5 & 3,2 & $\times 2,4$ \\
Zone de mesure 2 & 6,8 & 33,0 & 2,1 & 13,9 & $\times 4,3$ \\
Zone de mesure 3 & 0,9 & 5,2 & 0,1 & 1,2 & \\
\hline
\end{tabular}

Tableau 5. Rugosités mesurées avec les deux formats de description de la trajectoire.

\begin{tabular}{cccccc}
\hline & \multicolumn{2}{c}{ G1 } & \multicolumn{2}{c}{ B-spline Siemens } & \multirow{2}{*}{ Évolution de $R z$ avec la Tripteor X7 } \\
\cline { 1 - 4 } Rugosité $(\mu \mathrm{m})$ & $R a_{\max }$ & $R z_{\max }$ & $R a_{\max }$ & $R z_{\max }$ & \\
\cline { 1 - 3 } Zone de mesure 1 & 4,1 & 17,2 & 1,9 & 10,6 & $-40 \%$ \\
Zone de mesure 2 & 6,8 & 33,0 & 4,8 & 27,8 & $-18 \%$ \\
\hline
\end{tabular}

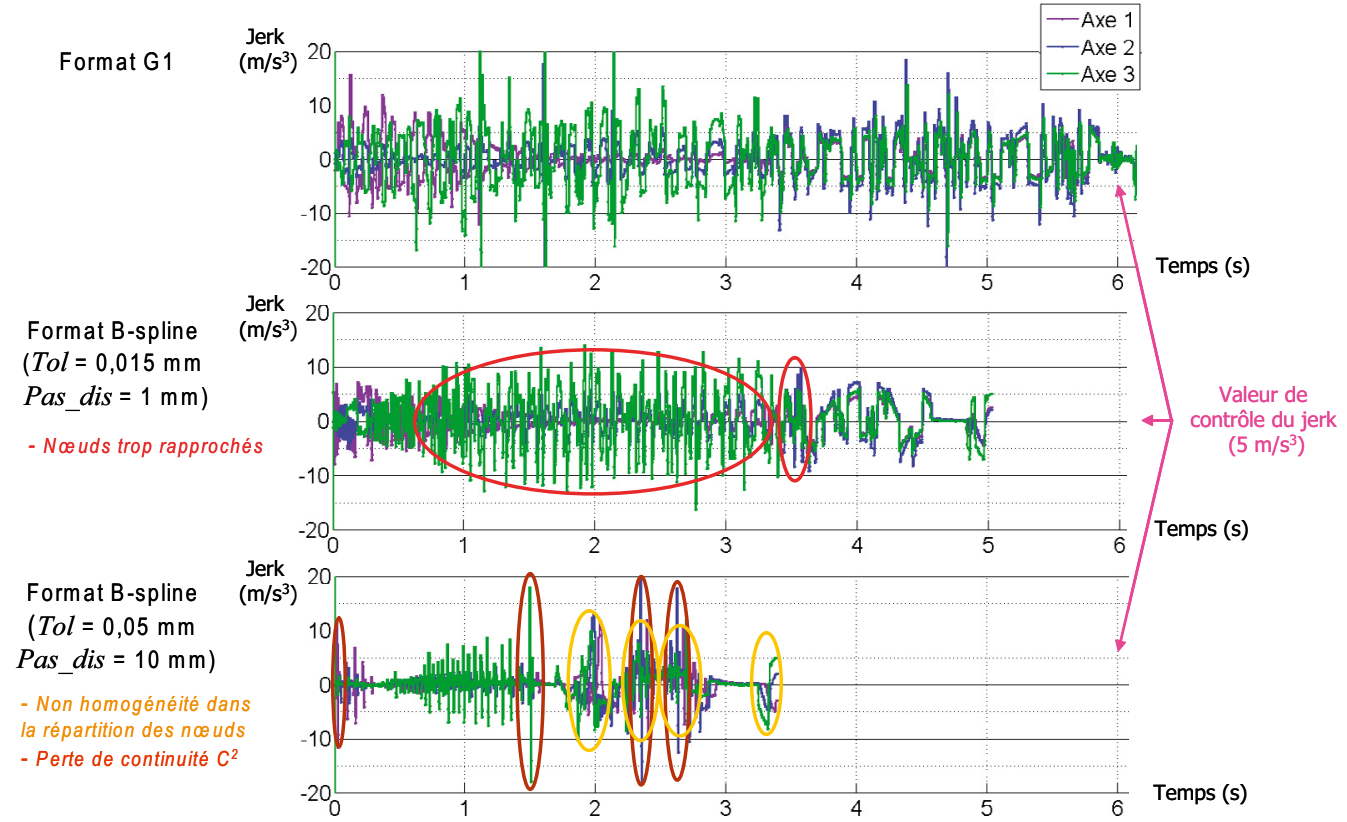

Fig. 5. Évolution du jerk des axes 1 à 3 pour les formats G1 et B-spline.

du $1^{\text {er }}$ mode propre de la structure n'est pas constante dans l'espace de travail opérationnel [15].

Après analyse des relevés de position des axes pour les deux formats, il s'avère que celui de la description Bspline, procurant un meilleur état de surface, atténue ces phénomènes perturbateurs provoqués par l'évolution du jerk et du snap (Fig. 5). Ces paramètres peuvent influencer le comportement de la commande et de la structure de la machine-outil. En effet, le traitement effectué par le DCN pour calculer l'évolution des déplacements des axes dépend d'abord du nombre de raccordements de B-spline le long de la trajectoire qui provoquent d'éventuelles discontinuités en tangence et en courbure. Le calcul résulte également de la séquence nodale, caractérisée par le nombre et la répartition des nœuds qui peuvent aussi perturber le traitement du DCN.

Ainsi, une valeur faible pour les paramètres Tol et Pas_dis entraîne une répartition trop rapprochée des nouds, tandis qu'une valeur plus élevée fait apparaître des raccordements de B-spline et une non-homogénéité dans la répartition des nouds. Ces caractéristiques ont des répercussions sur le traitement effectué par le DCN au niveau de l'évolution du jerk des axes le long de la trajectoire qui sont à l'origine de vibrations de la structure (Fig. 5).

Finalement, pour obtenir un meilleur état de surface, il serait intéressant d'étudier l'évolution de la position des axes pour de nouveaux traitements de la trajectoire tels qu'une interpolation B-spline de la trajectoire programmée ou un lissage de la consigne de déplacement des axes les plus dynamiquement sensibles $[16,17]$. Ces outils permettent effectivement d'améliorer le comportement dynamique de la machine-outil et par conséquent l'état de surface des pièces usinées.

Une augmentation de la productivité des opérations d'ébauche 2 axes $1 / 2$ peut aussi apparaître avec un posage cinématiquement optimal de la pièce en qualifiant les performances cinématiques de la machine-outil à structure parallèle à l'aide d'un indice de manipulabilité [18]. La valeur de cet indice varie suivant la pose de l'outil 
Tableau 6. Performances cinématiques de la PCI Tripteor X7 en fonction du posage de la pièce.

\begin{tabular}{|c|c|c|c|}
\hline & Posage 1 (initial) & Posage 2 & Posage 3 \\
\hline $\begin{array}{l}\text { Visualisation du polygone des } \\
\text { performances cinématiques } \\
\text { considéré constant dans le volume de } \\
\text { travail occupé pour l'usinage de la } \\
\text { pièce test }\end{array}$ & $\begin{array}{l}+ \text { Polygone de } \\
+ \text { performance } \\
+\end{array}$ & $\begin{array}{c}\text { Polygone de } \\
\text { Polygone } \\
\text { unité }\end{array}$ & \\
\hline Valeur de l'indice de manipulabilité & 14,32 & 7,25 & 53,72 \\
\hline Temps d'usinage d'ébauche & $517 \mathrm{~s}$ & $560 \mathrm{~s}$ & $483 \mathrm{~s}$ \\
\hline
\end{tabular}

dans l'espace de travail cartésien de la machine-outil, et permet de qualifier le gain cinématique existant entre les déplacements des axes et le déplacement, considéré plan, de l'outil. Ainsi, plus l'aire du polygone des performances cinématiques est grand, plus le gain est important et plus le suivi des trajectoires sera dynamique [19].

Un gain de productivité allant jusqu'à $14 \%$ peut alors être obtenu entre deux posages différents de la pièce (Tab. 6). Toutefois, l'indice retenu (calculé au centre du volume de la pièce brute) considère des déplacements d'outils uniformément répartis dans toutes les directions du plan de la table pour évaluer les performances cinématiques. Ce choix permet un calcul simple de l'indice, mais il n'est pas le mieux adapté aux trajectoires d'ébauches 2 axes $1 / 2$ de la pièce test. Il est alors intéressant de proposer une méthode de définition de cet indice en fonction des trajectoires (stratégie d'ébauche, direction de déplacement privilégiée, etc.).

\section{Conclusion}

Cette étude comparative permet de mettre en évidence les performances de la machine-outil à structure parallèle PCI Tripteor X7 vis-à-vis de l'usinage d'une pièce aéronautique à fort taux d'enlèvement de matière. Cette machine-outil offre une productivité accrue par rapport à une machine-outil à structure sérielle, une qualité dimensionnelle et géométrique générée satisfaisante pour l'application retenue, mais une qualité d'état de surface non acceptable vis-à-vis des exigences de qualité du domaine aéronautique.

L'étude met en avant l'influence du facteur d'amplification cinématique et mécanique de sa structure plus complexe sur la productivité, la qualité dimensionnelle, mais surtout sur les états de surface obtenus. Ainsi, pour exploiter au mieux les performances offertes par les machines-outils à structure parallèle, il faut mener un travail supplémentaire de définition du processus d'usinage, dédié à la machine. Cet article propose alors une première voie d'amélioration du comportement mécanique de la structure de la machine-outil (à l'origine des défauts d'état de surface) en modifiant le format de description de la trajectoire d'usinage qui influence le calcul des consignes de déplacement des moteurs des axes effectués par la CN. Cet article présente également une méthode d'amélioration de la productivité s'appuyant sur la détermination d'un posage de la pièce cinématiquement optimal.

Ces méthodes permettent d'améliorer les performances des machines-outils à structure parallèle, mais doivent être élargies à l'adaptation de l'ensemble des paramètres du processus d'usinage dont dépendent les performances d'accessibilité, de qualité et de productivité de façon à produire des pièces de structure aéronautiques conformes à la qualité requise avec une productivité maximale. Il est alors nécessaire d'identifier tous les paramètres influençant le comportement spécifique de ces machines-outils, puis de mettre en place des méthodes d'adaptation du processus s'appuyant sur des modèles suffisamment prédictifs du comportement, de façon à exploiter au mieux leurs performances.

Remerciements. Ce travail a bénéficié d'une aide de l'Agence Nationale de la Recherche (référence ANR-08-JCJC-0038-01). Ces travaux de recherche s'inscrivent dans le cadre de la fédération de recherche TIMS/CNRS 2856 financée par le conseil régional d'Auvergne, le ministère de la recherche, le CNRS et le CEMAGREF. Ce travail est conduit sous l'égide du groupe de travail Manufacturing 21 qui comprend 17 laboratoires français. Les thèmes abordés sont : modélisation $\mathrm{du}$ processus de fabrication, optimisation des conditions opératoires, fabrication virtuelle, validation expérimentale, nouvelles méthodes de fabrication. Les auteurs remercient la société PCI-SCEMM pour sa collaboration et le CETIM pour sa participation au sein du projet CAIUS.

\section{Références}

[1] M. Terrier, Optimisation du processus de fabrication en usinage à grande vitesse sur machines-outils à structure parallèle, Thèse de doctorat en Génie Mécanique, École Centrale de Nantes, 2005

[2] G. Pritschow, C. Eppler, T. Garber, Influence of the dynamic stiffness on the accuracy of PKM, 3rd Chemnitz parallel kinematic seminar, Chemnitz, Germany, 2002, pp. 313-333

[3] A. Hertel, Requirement for parallel kinematics for powertrain manufacturing in the automotive industry, 3rd Chemnitz parallel kinematic seminar, Chemnitz, Germany, 2002, pp. 753-762 
[4] N. Hennes, Ecospeed - an innovative machinery concept for high performance 5 axis machining of large structural components in aircraft engineering, 3rd Chemnitz parallel kinematic seminar, Chemnitz, Germany, 2002, pp. 763774

[5] K.E. Neumann, Exechon Concept - Parallel Kinematic Machines in Research and Practice (PKS'2006), Chemnitz, Germany, 2006, pp. 787-802

[6] T. Bonnemains, Étude du comportement mécanique des machines outils à structure parallèle en usinage grande vitesse, Thèse de doctorat, Université Blaise PascalClermont II, 2009

[7] M. Geldart, P. Webb, H. Larsson, M. Backstrom, N. Gindy, K. Rask, A direct comparison of the machining performance of a varix 5 axis parallel kinematic machining centre with conventional 3 and 5 axis machine tools, Int. J. Mach. Tools Manuf. 43 (2003) 1107-1116

[8] J. Angeles, Fundamentals of Robotic Mechanical Systems, Springer-Verlag, ISBN: 0-387-95368-X, 1997

[9] J. Tlusty, J. Ziegert, S. Ridgeway, Fundamental comparison of the use of serial and parallel kinematics for machine tools, Annals of CIRP 48 (1999) 351-356

[10] H. Chanal, E. Duc, J.Y. Hascoët, P. Ray, Reduction of a parallel kinematics machine tool inverse kinematics model with regard to machining behaviour, Mech. Mach. Theory 44 (2009) 1371-1385

[11] J.M. Langeron, E. Duc, C. Lartigue, P. Bourdet, A new format for 5 axis tool path computation using Bspline curves, Computer Aided Design 36 (2004) 1219-1229
[12] K. Erkorkmaz, Y. Altintas, High speed CNC system design. Part I: jerk limited trajectory generation and quintic spline interpolation, Int. J. Mach. Tools Manuf. 41 (2001) $1323-1345$

[13] U. Bravo, O. Altuzarra, L.N. López de Lacalle, J.A. Sánchez, F.J. Campa, Stability limits of milling considering the flexibily of the workpiece and the machine, Int. J. Mach. Tools Manuf. 45 (2005) 1669-1680

[14] S. Seguy, G. Dessein, L. Arnaud, Surface roughness variation of thin wall milling, related to modal interactions, Int. J. Mach. Tools Manuf. 48 (2008) 261-274

[15] R. Bearee, Prise en compte des phénomènes vibratoires dans la génération de commande des machines-outils à dynamique élevée, Thèse de doctorat en Automatique, ENSAM Lille, 2005

[16] V. Pateloup, E. Duc, P. Ray, Bspline approximation of circle arc and straight line for pocket machining, Computer Aided Design 42 (2010) 17-827

[17] C. Castagnetti, E. Duc, P. Ray, The domain of Admissible Orientation concept: A new method for five axis tool path optimisation, Computer Aided Design 40 (2008) 938-950

[18] J.P. Merlet, Les robots parallèles, Hermès, ISBN : 286601-599-1, 1997

[19] T. Kim, S.E. Sarma, Tool path generation along directions of kinematic performance: a first cut at machine optimal paths, Computer-Aided Design 34 (2002) 453468 